\title{
Adaptive identification and characterization of polar ionization patches
}

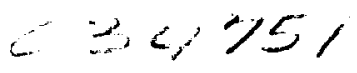

\author{
W. R. Coley and R. A. Heelis \\ William B. Hanson Center for Space siences, University of Texas at Dallas, Richardson
}

\begin{abstract}
Dynamics Explorer 2 (DE 2) spacecraft data are used to detect and characterize polar cap "ionization patches". loosely defined as large-scale $(>100 \mathrm{~km})$ regions where the $F$ region plasma density is significantly enhanced ( $\geq 100 \%$ ) above the background level. These patches are generally believed to develop in or equatorward of the dayside cusp region and then drift in an antisunward direction over the polar cap. We have developed a flexible algorithm for the identification and characterization of these structures, as a function of scale-size and density enhancement, using data from the retarding potential analyzer, the ion drift meter, and the langmuir probe on board the DE 2 satellite. This algorithm was used to study the structure and evolution of ionization patches as they cross the polar cap. The results indicate that in the altitude region from 240 to $950 \mathrm{~km}$ ion density enhancements greater than a factor of 3 above the background level are relatively rare. Further, the ionization patches show a preferred horizontal scale size of $300-400 \mathrm{~km}$. There exists a clear seasonal and universal time dependence to the occurrence frequency of patches with a northern hemisphere maximum centered on the winter solstice and the 1200-2000 UT interval.
\end{abstract}

\section{Introduction and Review}

While most emphasis in the study of high-latitude $F$ region irregularities has been on small-scale structures with scale sizes of a few meters to $10 \mathrm{~km}$, large-scale $(>10 \mathrm{~km})$ patches of enhanced plasma have also been observed in the auroral zone and polar cap $F$ region using a number of techniques. As more has been learned about the small-scale $(<10 \mathrm{~km})$ irregularities, a relationship with the larger structures has begun to emerge. Tsunoda [1988] observed that the phenomenology of the large-scale structures resembles that of the smaller-scale irregularities; that is, the irregularity source regions seem to occur in the vicinity of the larger-scale structures and the associated gradients required for the creation of plasma irregularities by convective plasma processes. This has stimulated an interest in the larger plasma fealures. The high-latitude large-scale plasma structures have been divided into four classes by Tsunoda [1988]: (1) polar cap "patches," (2) boundañ "blobs," (3) sunaligned arcs, and (4) auroral blobs. This paper deals with the first category, that of polar cap patches.

What is the precise definition oi a patch? There seems to be no general consensus, but instead, the definition varies according to the observer and the observational icchnique used. (Common grourd-based observational tcchniques include the use of riometers, photometers, ionosondes, all-sky cameras, and incoherent scatter radar.) One simple working definition might be the following: a patch is a limited region of increased plasma densi-

Copyright 1995 by the American Geophysical Union.

:aper number 95JA02700.

0148-0227/95/95JA-02700S05.00 ty in the polar cap that has horizontal dimensions of the order of $100-1000 \mathrm{~km}$. This definition is necessarily rather loose, since there is currently no agreement on the size of the density increase that constitutes a patch or, indeed, how to determine the background density level of the polar cap.

Patches were first recognized in the winter central polar cap using optical and radio wave diagnostic measurements [ Hill, 1963; Buchau et al, 1983; Weber et al., 1984]. These observations indicated nonlocally produced regions of $F$ region density that were a factor of 5-10 times above the background with maximum electron densities of $\sim 10^{6} \mathrm{~cm}^{-3}$ inside the patches. Weber et al. [1986] used the Global Positioning System satellites to determine the line of sight total electron content (TEC). They found patches with an increase in TEC of a factor of 3-4 which correlated well with all-sky images of patches taken at $6300 \AA$. Patches are not stationary. Incoherent scatter radar measurements from Sondrestrom [Kelley and Vickery, 1984] have shown patches convecting poleward through the cusp region into the polar cap. Similar results have come from dayside observations using the Chatanika radar [Foster and Doupnik, 1984]. Weber et al. [1986] have tracked patches convecting antisunward through the polar cap over distances of $3000 \mathrm{~km}$.

Several observers have described characteristics that they have found are commonly associated with patches [Buchau et al., 1983; Weber et al., 1984, 1986; Roger et al., 1994]. The following list indicates both arcas of agreement and disagreement:

1. Patches are regions of enhanced 030 -nm emission.

2 . Patches drift antisunward across the polar cap at the $E \times B$ convection velocity.

3. Weber et al. [1984] believe patches to be a winter phenomenon, occurring when the $z$ component of the 
IMF $\left(B_{z}\right)$ is negative and $K p \geq 3$. Roger et al [1994], on the other hand, have examined data from the Polar Anglo-American Conjugate Experiment (PACE) and concluded there is no significant seasonal variation in the occurrences of patches.

4. Scintillation measurements indicate that patches are often accompanied by intermediate-scale $(1-10 \mathrm{~km})$ irregularities [Weber et al, 1984]. While these irregularities are often present throughput the entire patch, there is a tendency for them to be prevalent on the trailing edge.

5. No energetic particle precipitation occurs in the patch while in the polar cap.

6. Baker et al. [1989] have observed the simultaneous formation of patches in geomagnetically conjugate regions.

The generation mechanism for polar cap patches remains an especially controversial topic with several different suggested scenarios. One idea suggests that $1000 \mathrm{~km}$ pieces of solar-euv produced plasma are, occasionally, detached from the dayside subauroral ionosphere in the winter hemisphere through the development of a large, eastward electric field [Tsunoda, 1988]. These occasions probably occur when the $B_{z}$ or $B_{y}$ component of the IMF changes rapidly. A southward turning $B_{z}$ leads to the expansion of the auroral oval and the development of a strong two-cell convection pattern which leads to antisunward flow in the (formerly subauroral) region of solar-enhanced plasma. As this plasma is drawn into the polar cap it is detached from the following plasma by a northward turning of $B_{2}$ thus becoming a patch. Anderson et al. [1988] have modeled this mechanism and successfully duplicated incoherent scatter radar measurements at Thule, Greenland. In this model the patch formation region lies equatorward of the dayside cusp, and patch formation depends on the presence of relatively large plasma concentration gradients lying close to the sunward edge of the convection boundary. The gradient results from the close proximity of the solar terminator. This approach has been extended by Sojka et al. [1993] and Decker et al. [1994] to show how large-scale plasma structures may arise from time-dependent convection processes.

There is some evidence that patches are formed in the ionospheric footprint of the cusp [Baker et al., 1989; Roger et al., 1994]. Roger et al. [1994] describes a mechanism whereby patches are formed in the cusp region during socalled "flow channel events" (FCE). An FCE consists of a short-lived plasma jet with a speed of $\sim 2 \mathrm{~km} \mathrm{~s}^{-1}$. A simultaneous change in the $B_{y}$ component of the IMF is also required. A region of enhanced plasma density in the cusp due to electron precipitation is isolated by a FCE which causes a local plasma density depletion. Both $B_{z}$ and $B_{y}$ are initially negative. Immediately afterward $B_{y}$ changes toward zero causing the plasma flow to have a more poleward direction. The newly formed patch then drifts antisunward into the polar cap. This model does not require the formation region to be in the winter hemisphere. Indeed, it allows for the formation of patches in geomagnetically conjugate regions.

While spacecraft have been used to investigate small to mid sized $(<150 \mathrm{~km}$ ) ionospheric density structures [Sagalyn et al., 1974; Villain et al., 1986], relatively little work has been done to date on the study of large-scale polar cap ionization density enhancements detected through the use of satellite data. We call these enhancements "ionization patches" in order to differentiate then from structures (also called patches) measured by other techniques. The Dynamics Explorer 2 spacecraft represents a large, almost untouched, resource that is eminently suitable to answer many of the unsolved questions regarding the origin, description, and evolution of polar cap ionization patches. This paper describes tools that utilize this data set and provide a more accurate picture of the structure of polar cap ionization patches.

\section{Observational Database}

The database we used was provided by the retarding potential analyzer (RPA), ion drift meter (IDM), and the langmuir probe aboard the DE 2 spacecraft. These instruments are described in detail by Hanson et al. [1981], Heelis et al. [1981], and Krehbiel et al [1981]. These instruments provide ion density, ion and electron temperatures, and plasma flow velocity. The lifetime of the satellite covers the period from August 1981 through February 1983, a time of high solar activity with a mean $F_{10.7}$ of about 190 . The $90^{\circ}$ inclination of the orbit causes the local time of the ascending node to traverse all local times in about 1 year. Thus local time and season are locked together in a manner fixed by the launch date and time. For the DE 2 database, passes across the dawn and dusk local time sectors are taken either in the summer or the winter months. Passes near local noon or midnight are taken during the equinoxes. However, at latitudes above $75^{\circ}$ magnetic, the motion of the magnetic pole about the geographic pole provides good local time coverage in all seasons.

\section{Patch Identification Technique} Patch Identification Technique
We desire to characterize the large-scale density fea-
tures in the polar cap as a function of scale-size, density enhancement, and other parameters. The first task is the development of a functional definition of an ionization patch capable of selecting suitable features from the total ion density measurement of the RPA. Several questions arise: (1) How is the edge of a patch determined?, (2) What is the minimum density enhancement defining a patch?, (3) How is the background density level determined?, and (4) What are the minimum and maximum horizontal scale-sizes that define a patch? Figure 1 shows a schematic diagram of a simple density enhancement that might be encountered during a polar pass of DE 2 .

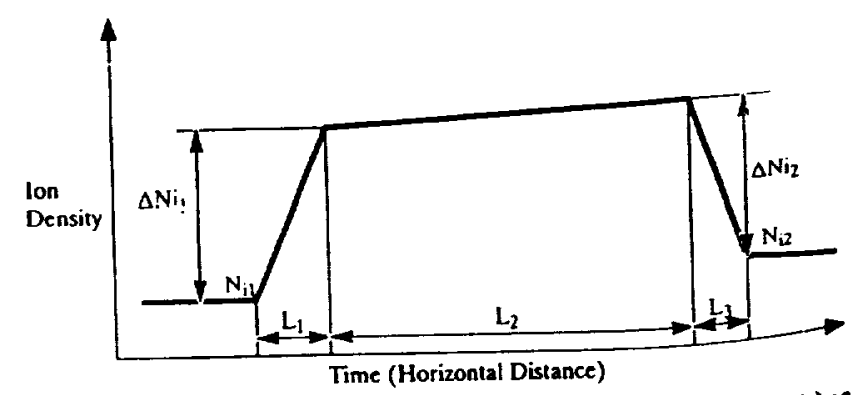

Figure 1.Schematic diagram of a densityenhancement (patch) as seen by a satellite traversing the polar cap. 
- It illustrates some of the parameters that are used here to characterize an ionization patch: $L_{1}$ and $L_{3}$, the horizontal distances neer which the number density changes from the background levels ( $N_{i 1}$ and $N_{i 2}$ ) to the internal density of the patch, $L_{2}$, the horizontal size of the patch along the spacecraft trajectory, and $\Delta N i_{1}$ and $\Delta N i_{2}$, the magnitudes of the density enhancements at the opposite sides of the patch. In order to answer the first and second questions above it is necessary to examine the DE 2 database for features that fall into different categories dependent on the parameters describing a patch. Largescale $(\geq 100 \mathrm{~km})$ ionization structures in the $F$ region may be seen in RPA data from the DE 2 spacecraft by recognizing the occasions when the total ion concentration is enhanced by either a given absolute amount $\left(\Delta N_{i}\right)$ or by a relative factor $\left(\Delta N_{i} / N_{i}\right)$ above a background level. The edges of these enhancements (ionization patches) may be determined by specifying a horizontal extent over which the ion conaentration must rise by some given minimum percentage above the background level (i.e. specifying a minimum gradient over a given horizontal distance). To qualify as a patch, a density enhancement must have edges that meet a gradient criterion and a maximum density that meets either a relative or an absolute enhancement criterion.

The last two questions above are related. The choice of a maximum horizontal scale-size for a patch means that features somewhat larger than this may be considered as background. In addition, features smaller than the chosen minimum horizontal size can be considered as noise (from the point of view of patch identification) and then removed. We have chosen to accomplish these tasks through the use of a median filter algorithm. Such a filter can directly remove features smaller than the desired minimum scale-size. By applying the filter to the data at the maximum scale-size, a background level is determined that can then be subtracted from the original data. By applying the two procedures sequentially, the net result is a bandpass filter that will only leave features in the desired range of horizontal sizes.

We selected the DE 2 RPA database for this study as it provides an extensive on-line data set that is suitable for doing both case studies and statistics. The RPA and IDM instruments provide plasma density, velocity and ion temperature information while the langmuir probe provides electron temperature data that will help to differentiate the auroral zone, cusp, and polar cap regions by indicating $T_{e}$ enhancements due to precipitating energetic electrons. The top two panels of Figurc 2 present the ion density, electron temperature, and ion temperature profiles for a dusk-dawn northern hemisphere polar pass of the DE 2 spacecraft. Note the high degree of structure in the ion density at all scale-sizes down to the approximately $7.5 \mathrm{~km}(1 \mathrm{~s})$ resolution of the data. The saw-tooth profile in the ion density is due to the lack of appropriate calibration from different sweep modes of the RPA, but does not affect identification of structures with scale sizes greater than $50 \mathrm{~km}$ or so. We see four polar cap ion density enhancements marked $\mathrm{E} 1-\mathrm{E} 4$ in Figure 2a that might possibly be identified as patches.

Figure $2 \mathrm{c}$ illustrates the median filter process applied to the ion density trace of the DE 2 polar pass of Figure 2a using some reasonable values for the tests. We have applied the scale-size filter to pass features in the $100-875 \mathrm{~km}$ size range. Note that the density scale is now linear and shows the ion density relative to the subtracted baseline. The density enhancements E1-E4 are still present if somewhat less prominent. For the purposes of this plot we identify the edge of a patch by a density increase of at least $40 \%$ over the background value in a horizontal distance of $140 \mathrm{~km}$. Feature E4 fails this test at both sides. Feature E1 has only a trailing edge. E2 and $\mathrm{E} 3$ both have a leading and trailing edge and thus pass the edge gradient test. Next if we require, for example, that the peak ion density in a patch must exceed the background density by at least $100 \%\left(\Delta N_{i} / N_{i} \geq 1\right)$, then only feature E2 satisfies this test with a value for $\Delta N_{i} / N_{i}$ of 1.09. The solid vertical line indicates the density increase associated with $\mathrm{E} 2$ (leading edge) that meets the edge criterion. The dashed vertical line indicates the density decrease (trailing edge) that meets the edge criterion. This ionization patch has a horizontal size of $258 \mathrm{~km}$. The middle panel on Figure 2 shows the RPAdetermined ion temperature and the electron temperature measured by the on-board langmuir probe. These temperatures are useful in determining whether the density enhancements were locally generated by precipitating particles (indicated by a temperature enhancement) or convected to their current locations. In the current example the depression in electron temperature indicates that this density enhancement was not locally generated and probably $E \times B$ drifted from the sunward direction.

\section{Results}

With such an automated identification process we have examined the entire DE 2 RPA-derived ion density database to determine the distribution of spatial structures (patches) with different horizontal scale sizes and different magnitudes of density variation. We have chosen to use an edge criterion of a minimum $40 \%$ increase in $140 \mathrm{~km}$ horizontal distance to examine Northern hemisphere structures in the $100-1250 \mathrm{~km}$ horizontal scale-size range. Figure 3 shows the distribution of ionization patches as a function of the magnitude of patch enhancement. The altitude range over which these structures were measured was $240-950 \mathrm{~km}$. Above $70^{\circ}$ invariant latitude plasma structures with magnitudes that $\mathrm{ex}$ ceed the background level by up to a factor a 8.5 can be identified. The number of structures increases exponentially with decreasing scale size and structures with horizontal scale sizes greater than $100 \mathrm{~km}$ and density enhancements exceeding a factor of 2 are quite rare relative to the smaller scale size structures. Examining the DE 2 database in this region without regard to IMF the total number of ionization structures observed that meet the edge criterion and where the patch density exceeds the background by $100 \%$ or more is 281 . It should be noted that these data are appropriate to a solar maximum period when the average $10.7-\mathrm{cm}$ solar flux is about 180 .

Since many current theories of patch formation and evolution require antisunward convection across the polar cap (negative $B_{z}$ ) we have chosen to examine the ionization patches by separating the results according to hourly averages of the IMF. For the purposes of estab- 

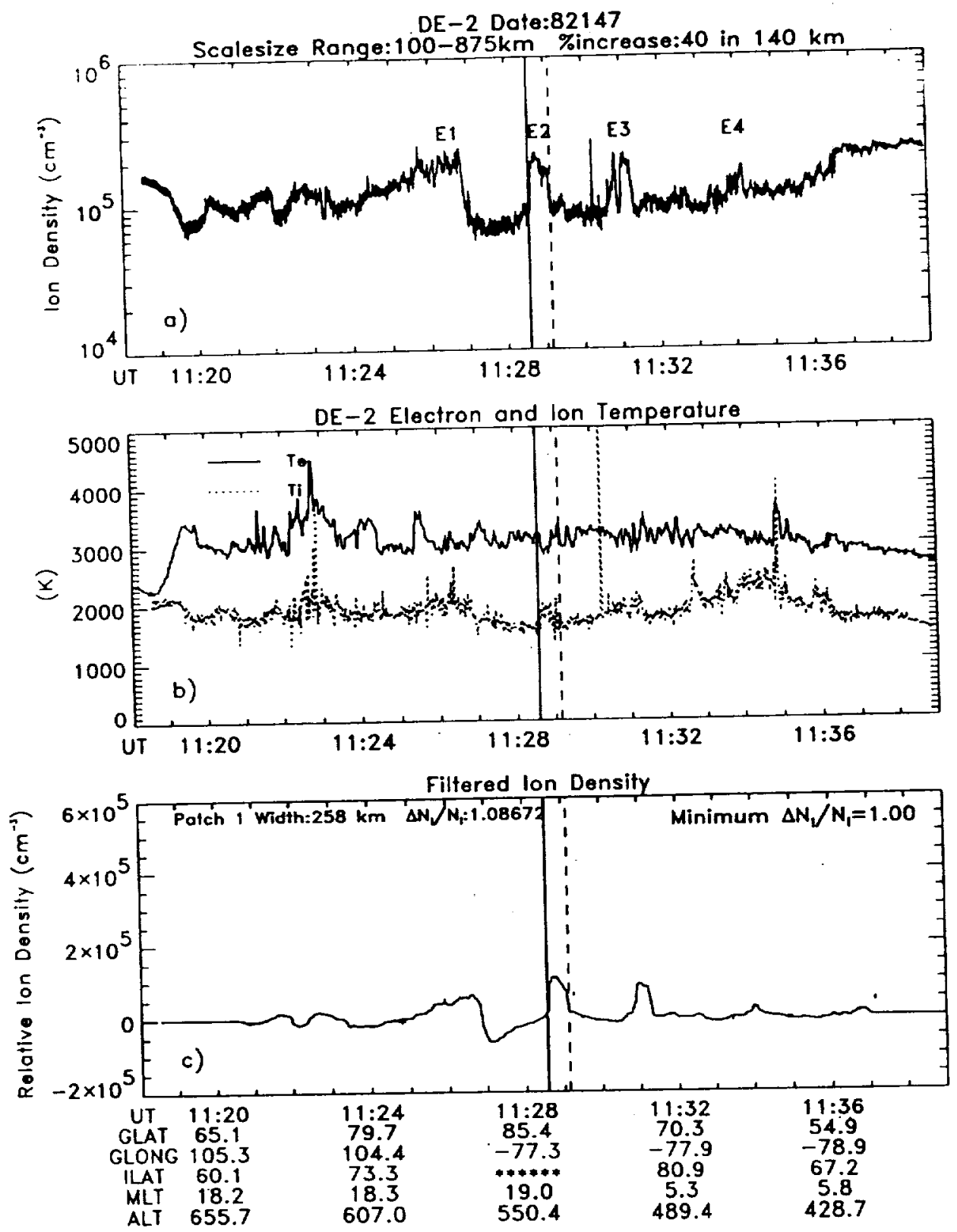

Figure 2. (a) DE 2ion density for a typical polar pass. Density enhancements E1 - E4 represent possible ionization patches. (b) Ion and electron temperatures from the RPA and Langmuir probe, respectively. (c) Linear plot of the median filtered ion density with background subtracted. The vertical solid and dashed lines show the leading and trailing edges of $E 2$, the only enhancement to meet all the criteria used to define an ionization patch (see text).

lishing trends in the patch characteristics we have selected only those features with enhancements greater than $100 \%$. The use of hourly IMF values does not allow specific temporal variations in this parameter to be identified with patch occurrence. However, it will provide a first order measure of the sensitivity of patch occurrence frequency to the global characteristics of the convection pattern.

Figure 4 a shows the distribution of horizontal scale sizes of ionization patches along the satellite track that are observed for the observed 131 cases with enhancements occurring when $B_{z}<0$. Recalling that structures of less than $\sim 100 \mathrm{~km}$ and more than $\sim 1250 \mathrm{~km}$ are filtered out, there is clearly a preferential scale size between
300 and $400 \mathrm{~km}$ with a secondary peak at $600-700 \mathrm{~km}$. Figure $4 \mathrm{~b}$ indicates that the distribution of horizontal scale sizes is quite similar when data representing all values of the IMF are used. The most notable difference is the decrease in the size of the secondary peak at $600-700 \mathrm{~km}$ scale size. In addition, further examinaticn of the data reveals that the observed scale sizes of ionization patches do not depend on the path of the satellite with respect to the dawn-dusk meridian, and therefore we conclude that the observed structures are either nominally circular in shape or, if elongated, the orientation of the elongation is random.

Figure 5 a shows the number of ionization structures with enhancements larger than $100 \%$ observed as a func- 



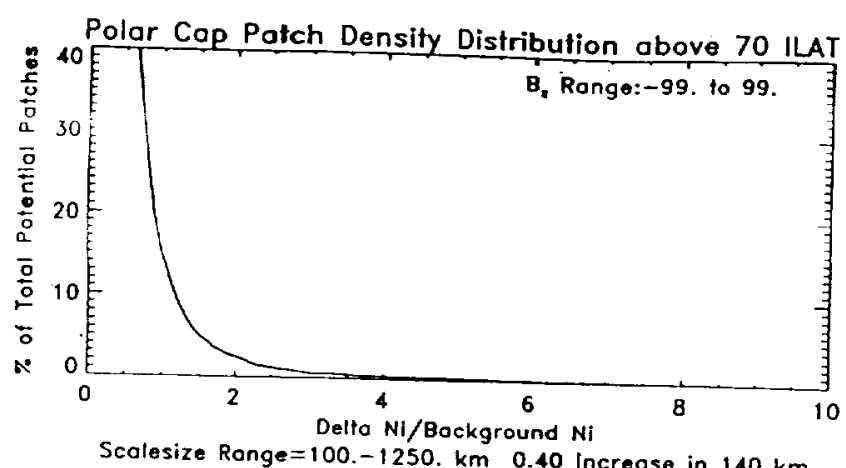

Figure 3. Percentage of northern hemisphere density enhancements larger than a given value. All values of $B_{z}$ were allowed.

tion of the month of the year for conditions of negative $B_{z}$. Local time and season are locked together in the DE 2 polar orbit, but at magnetic latitudes above $75^{\circ}$ the offset of the geographic and geomagnetic poles assure reasonable coverage in local time at all seasons. These structures at all scale sizes show a strong seasonal preference for formation in the winter when polar cap ionospheric densities are low. This seasonal dependence perhaps indicates that the source mechanism of the structure is only effective in the absence of substantial ionization production from the Sun. One might wonder whether this is only an apparent seasonal dependence since, due to the increased ion density in the summer polar cap, ionization enhancements of a given absolute $\Delta N_{i}$
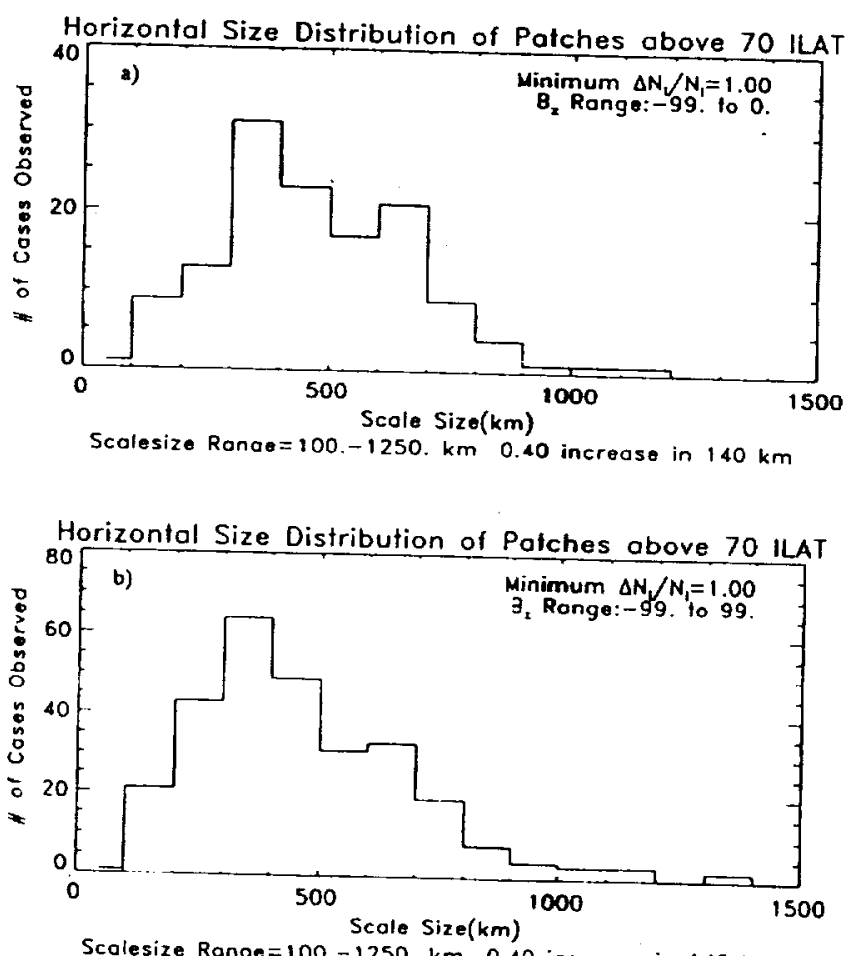

Figure 4. (a)Size distribution of the 131 ionization patches with density enhancements of at least $100 \%$ and negative $B_{z}$. (b) Size distribution of the 281 ionization patches with density enhancements of at least $100 \%$. All values of the interplanetary magnetic fistc (IMF) are included.


Figure 5. (a) Occurrence frequency of ionization patches versus month. Month 0 and month 12 are both December. Only data for which the $z$ component of the $\mathrm{IMF}$ is negative are used. (b) Occurrence frequency of ionization patches versus month. Month 0 and month 12 are both December. All values of the IMF are included.

would not pass the $\Delta N_{i} / N_{i}$ criterion in summer. However, it was found that the patch statistics using an absolute $\Delta N_{i}$ enhancement criterion rather than a relative one produces no substantive difference in the seasonal morphology. From Figure $5 b$ it is seen, somewhat surprisingly, that there is also no apparent change in the seasonal distribution of ionization patches when data from positive values of the IMF are included. For cases of positive $B_{z}$ it might be suggested that a different production mechanism is active involving generation of ionization enhancements in the polar cap due to the energetic particle precipitation that creates polar cap arcs. However, examination of electron temperature structure on a case by case basis reveals no significant $T_{e}$ enhancements associated with the observed ionization patches. This would seem to indicate the possibility that the same mechanism may operate for both positive and negative $B_{2}$ to generate ionization patches with enhancements of $100 \%$ or greater.

For conditions of negative $B_{z}$ it is expected that, if patch formation occurs in or equatorward of the dayside cusp, the changes in the antisunward convection flow pattern will lead to different locations in the observation of dayside polar cap of ionization patches as the $B_{y}$ component of the IMF varies between positive and negative. Figures $6 \mathrm{a}$ and $6 \mathrm{~b}$ present the location of observed patches for conditions of negative $B_{z}$ with $B_{y}$ negative and positive, respectively. A minimum increase in ion density of $80 \%$ over background was used for this plot in order to improve the statistics. We see, particularly above $80^{\circ}$ ILAT for negative $B$, a larger number of patches in the afternoon sector between 1200 and 1800 


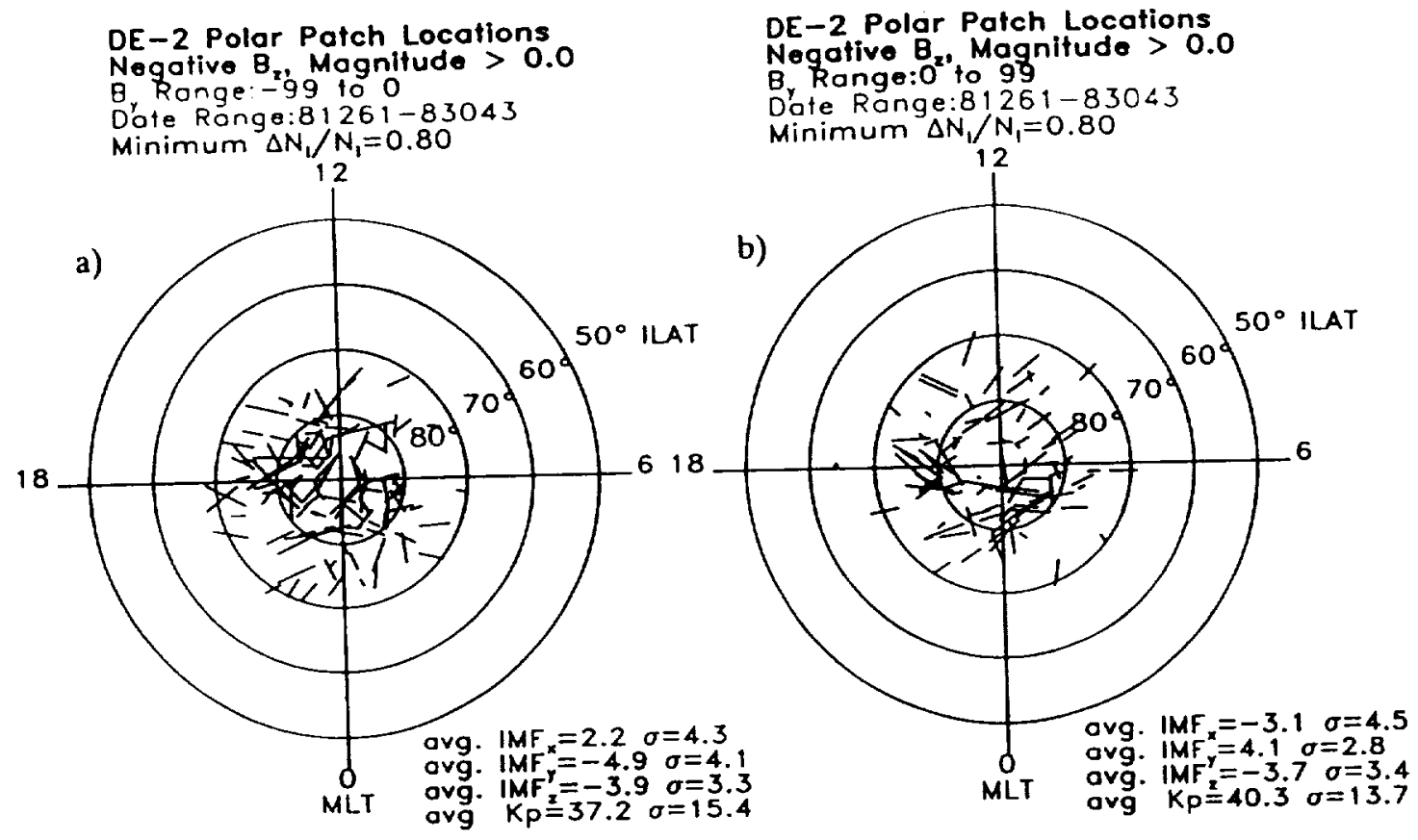

Figure 6. Location of polar ionization patches for conditions of negative $B_{z}$ and (a) negative $B_{y}$ and (b) positive $B$. The lines represent the sections of the spacecraft ground track where ionization patches are observed. The minimum ionization enhancement to qualify as a patch is $80 \%$ for this plot.

MLT than between 0600 and 1200 MLT. Conversely, in Figure $6 \mathrm{~b}$, we see the reverse pattern of more patches in the morningside. This is consistent with the shift of antisunward flow from the cusp region in a two cell convection pattern and indicates that formation of patches in or equatorward of the dayside cusp is at least plausible. Note also the lack of a spatial scale size dependence on the spacecraft track as mentioned earlier.

Further evidence of the role of the cusp is presented in Figure 7a which presents a contour plot of the occurrence frequency (number of patches per polar pass) of northern hemisphere ionization patches versus UT and month for $\Delta N_{i} / N_{i} \geq 1.0$ and $B_{z}<0$. In addition to the already noted seasonal dependency, we see a double peak maximum covering roughly the $1200-2000$ UT interval, with the most probable patch occurrence at $1800 \mathrm{UT}$. This is the time interval during which the ionospheric footprint of the cusp region extends to the lowest geographic latitude on the dayside, allowing solar produced ionospheric plasma to be introduced through this region into the polar cap [Foster, 1993]. In Figure $7 \mathrm{~b}, \Delta N_{i} / N_{i}$ $\geq 0.8$ and all IMF values were used, significantly increasing the patch occurrence frequencies, but leaving essentially the same UT dependence.

\section{Discussion}

The description of ionization patches given here applies to the plasma near and above the $F$ peak. It is thus relevant to the recent studies by Sojka et al. [1993] and Decker et al. [1994] that investigate the behavior of the $F$ region under the influence of variable convection patterns. We point out that $F$ region ionization patches with density enhancements of a factor of 5 or greater are extremely rare and thus the mechanisms involved in pro- ducing observed patches may be more subtle than those invoked in models to change the patch density by a factor of 10 or more. While depletions below a nominal background level may exist, they are not responsible for the apparent appearance of the patches described here. The observation of patch location and occurrence frequency clearly favor a plasma source on the dayside. The scale size used to establish the background density in this work, is in excess of $1250 \mathrm{~km}$ and is therefore a substantial fraction of the polar cap size. Thus the plasma enhancements studied here are evidently composed of plasma that would not convect into the polar cap in the absence of the patch mechanism. It therefore seems reasonable to assume that the source plasma resides in the sunlit atmosphere at lower latitudes outside the polar region and is delivered to higher latitudes in some time variable manner. This is consistent with the most frequent observation of patches during winter. In the absence of a mechanism to feed this plasma into the polar cap it will slowly rotate with the Earth. When the IMF is northward the convection pattern is usually restricted to higher latitudes and may involve substantial deviations from a two-cell configuration. Nevertheless, the lowest-latitude flows are usually sunward and rotate to antisunward near local noon. Thus an expansion and contraction of the pattern may serve to modulate the sunlit plasma flow into the high-latitude region. In order fo: the lower-latitude plasma to be delivered to the polar cap, the auroral zone flows must either expand, or be reoriented on rather short timescales. A preferred horizontal scale size of $300-400 \mathrm{~km}$, independent of the spacecraft flight path through the patch, may suggest a connection between the effective width of the delivery region to the polar cap and the temporal timescale over which the delivery is made. If this is the case then, for 
nominal polar cap potential drops of 50 to $100 \mathrm{kV}$, timescales of a few minutes are appropriate and consistent with those invoked by recent models [Decker ct al., 1994]. There is a dependence of patch location on IMF $B y$ providing some evidence to support the notion that ionization patches are southward IMF phenomena. However, it should be emphasized that patches can be observed when the IMF is positive. We have been unable to establish the relationship between patch appearance and the stability of the $z$ component of the IMF, and thus it may be possible that patches continue to be observed only shortly after a transition from south to north IMF but not when the IMF is steady northward and Sun-aligned arcs are most frequently observed. Examination of specific patch occurrences with higher temporal resolution of the IMF measurements would be required to resolve this issue. Until a more exhaustive study of ionization enhancements is completed it seems prudent not to rule out the existence of ionization patches when the IMF is northward.

It should be emphasized that substantial departures from the characteristics shown here and those derived from the observation of enhanced airglow emissions are possible. Enhanced airglow emission may be produced not only by an increase in the $\mathrm{O}^{+}$concentration but also by a lowering of the ionization layer to a region of higher $\mathrm{O}_{2}$ concentration. The role of neutral winds and $E \times B$ drifts in lowering and raising the $\vec{i}$ region may be important considerations in the formation of ionization and airglow patches, but they need not produce the same characteristics. These differences may be responsible for the very large contrasts in airglow intensity used to define a patch as opposed to the more modest density enhancements described here. While we may confidently associate the ionization patches described here with airglow enhancements, the association between airglow enhancements and $F$ region density increases may not be so readily made. This ambiguity points toward further correlative studies that should be undertaken to establish the relationships more precisely.

\section{Conclusions}

By examining the $F$ region polar cap ionosphere from measurements made on DE 2 we have provided a preliminary description of ionization patches. The framework used to define ionization patches is adaptive in that it allows a variety of different spatial scale sizes and relative magnitudes to be investigated. A characteristic spatial scale size in the range $300 \mathrm{~km}$ to $400 \mathrm{~km}$ is observed

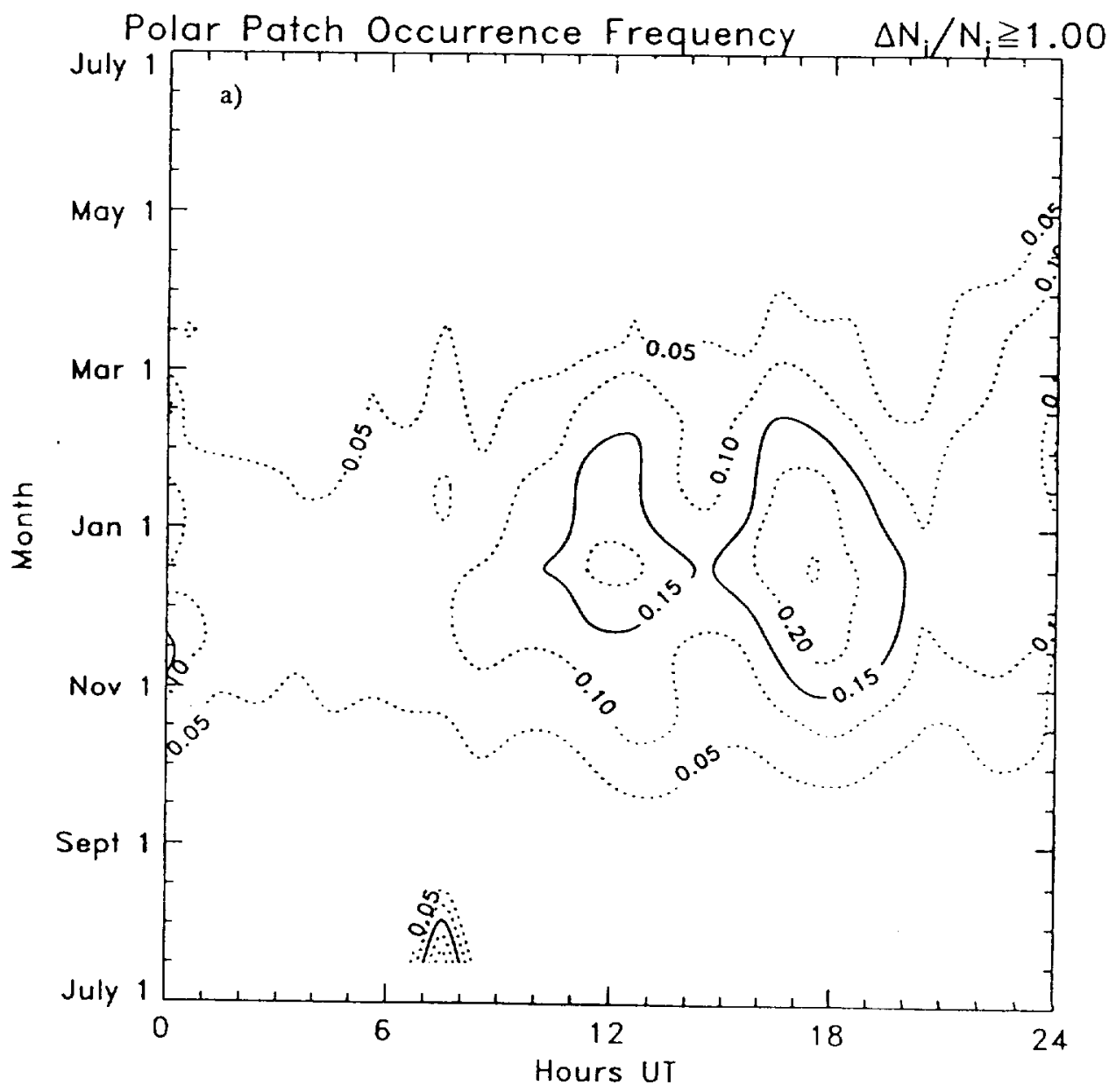

Figure 7. (a) Contour plot of the occurrence frequency of polar patches versus UT and month. Only ionization patches occur ring during periods of negative $B_{z}$ are used. (b) Contour plot of the occurrence frequency of polar patches versus UT and month. All ionization patches with an $80 \%$ increase in density over the background regardless of IMF value are used. 


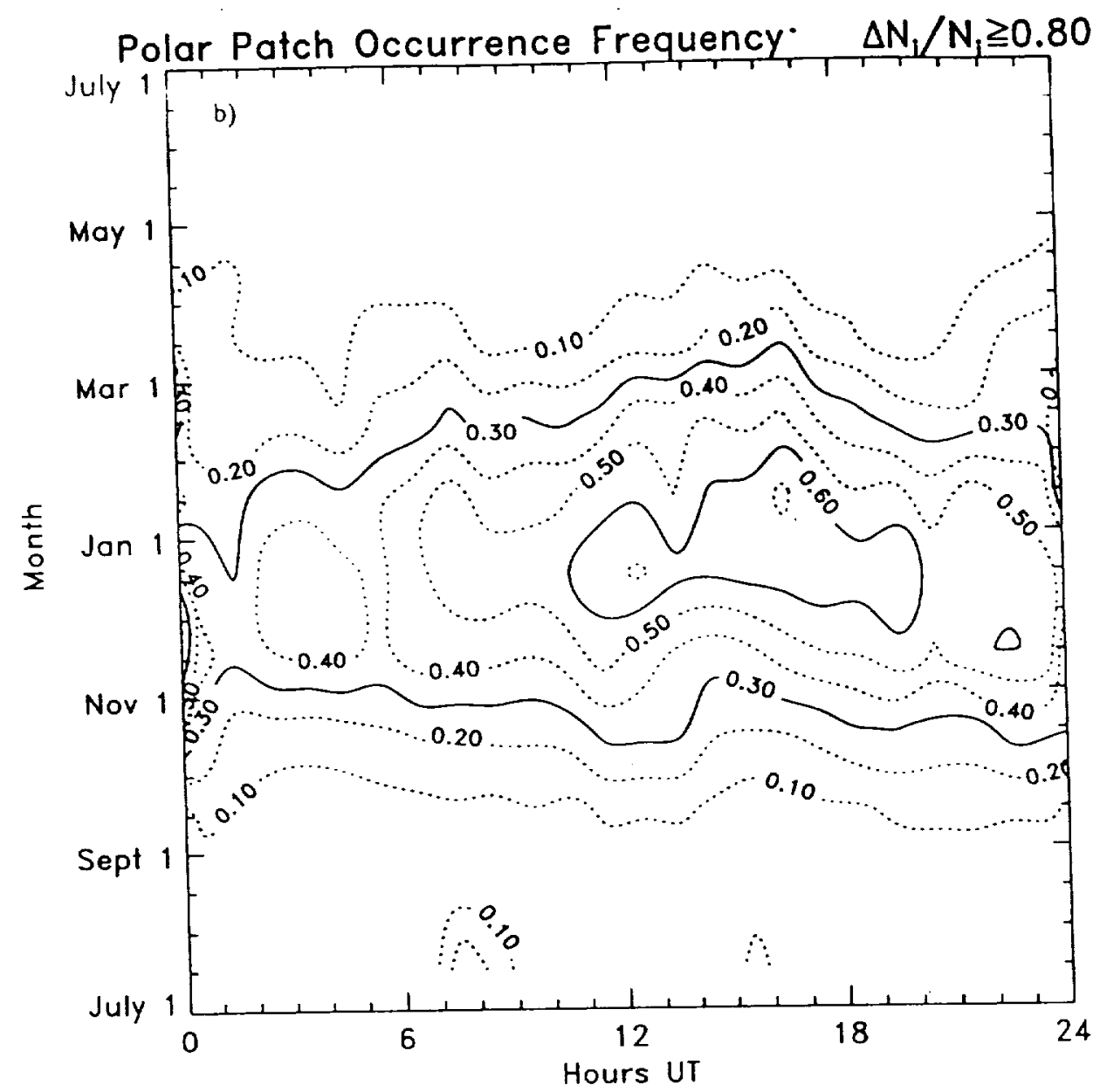

Figure 7. (continued)

to be independent of the path through a patch and reasonably independent of the magnitude of the patch enhancement, at least for enhancements greater than $80 \%$. We find that density enhancements larger than a factor of 5 are extremely rare and suggest that formation mechanisms capable of producing enhancements of $100 \%$ or so are the dominant feature of a time variable convection pattern. Observations are consistent with a source for the plasma equatorward of the cusp in a region that is slowly rotating with the Earth, with an entry into the polar cap that is temporally modulated by expansion of the polar cap or reconfiguration of the dayside auroral zone flows on timescales of a few minutes.

Acknowledgments. This work is supported at the University of Texas at Dailas by Air Force Geophysics Directorate contract F19628-93-K-0008, NationalAeronauticsand Space Administration grant NAGW-4442, and by National Science Foundation grant ATM-9311392.

The Editor thanks R. A. Greenwald and another referee for their assistance in evaluating this paper.

\section{References}

Anderson, D. N., J. Buchau, and R. A. Heelis, Origin of density enhancements in the winter polar cap ionosphere, Radio Sci., 23, 513, 1988.

Baker, K. B., R. A. Greenwald, J. M. Ruhoniemi, J. R. Dudeney, M. Pinnock, N. Mattin, and J. M. Leonard, PACE, Polar An-
glo-American conjugate experiment, Eos Trans. $A G U, 70,785$, 1989.

Buchau, J., B. W. Reinisch, E. J. Weber, and J. G. Moore, Structure and dynamics of the winter polar cap $F$ region, Radio $S c i$., 18, 995, 1983.

Decker, D. T., C. E. Vallardares, R. Sheehan, S. Basu, D. N. Anderson and R. A. Heelis, Modeling daytime $F$ layer patches over Sondrestrom, Radio Sci, 29, 249, 1994.

Foster, J. C., Storm time plasma transport at middle and high latitudes, J. Geophys. Res., 98, 1675, 1993.

Foster, J.C., and J.R. Doupnik, Plasma convection in the vicinity of the dayside cleft, J. Geophys. Res., 89, 9107, 1984.

Hairston, M. R., and R. A. Heelis, High-latitude electric field studies using DMSP data, Rep. PL-TR-93-2036, 45 pp., Phillips Lab., Hanscom Air Force Base, Mass., 01731-3010, 1993.

Hanson, W. B., R. A. Heelis, R. A. Power, C. R. Lippincott, D. R. Zuccaro, B. J. Holt, L. H. Harmon, and S. Sanatani, The retarding potential analyzer for Dynamics Explorer-B, Space Sci. Instrum., 5, 503, 1981.

Heelis, R. A., The effects of interplanetary magnetic field orien tation on dayside high-latitude ionosphericconvection, J. Geopliys. Res., 89, $2873,1984$.

Heelis, R.A., and M. R. Hairston, Studies of ionospheric dynamics utilizing data from DMSP, Rep. GL-TR-90-0047, 39 pp., Geophysics Lab., Hanscom Air Force Base, Mass., $01731-5000,1990$.

Heelis, R. A., W. B. Hanson, C. R. Lippincott, D. R. Zuccaro, L. H. Harmon, B. J. Holt, J. E. Doherty, and R. A. Power, The ion drift meter for Dynamics Explorer-B, Space Sci. Instrum., 5 , S11, 1981. 
Hill, J.R., Sudden enhancements of $F$ layer ionization in polar regions, J. Atmos. Sci, 20, 492, 1963.

Kelley, J D., and J T Y Sery, $F$-regionionospheric structure associated with anii-sunward flow near the dayside cusp, Geophys. Res. Lett., 11, 907, 1984.

Krehbiel, J. P., L. H. Brace, R. F. Theis, W. H. Pinkus, and R. B. Kaplan, The Dynamics Explorer Langmuir probe instrument, Space Sci. Instrum, 5, 493, 1981.

Robinson, R. M., R. T. Tsunoda, J. F. Vickrey, and L. Guerin, Sources of $F$-region ionization enhancements in the nighttime auroral zone, J. Geophys. Res., 90, 7533, 1985.

Roger, A. S., M. Pinnock, J. R. Dudeney, K. B. Baker, and R. A. Greenwald, A new mechanism for polar patch formation, $J$. Geophys. Res., 99, 6425, 1994.

Sagalyn, R.C., M.Smiddy, and M. Ahmed, High-latitude irregularities in the top side ionosphere based on Isis 1 thermal ion probe data, J. Geophys. Res. 79, 4252, 1974.

Sojka, J. J., M. D. Bowline, R. W. Schunk, D. T. Decker, C. E. Valladares, R. Sheehan, D. N. Anderson, and R. A. Heelis, Modeling polar cap $F$-region patches using time varying convection, Geophys. Res. Letr., 20, 1783, 1993.

Tsunoda, R. L., High-latitude $F$ region irregularities: A review and synthesis, Rev. Geophys., 26, 719, 1988.
Villain, J. P., C. Beghin, and C. Hanuise, ARCAD3-SAFARI coordinated study of auroral and polar $F$-region ionospheric irregularities, .1mn. Gcophls., 4, 61, 1956.

Weber, E. J., J. Buchau, J. G. Moore, J. R. Sharber, R. C. Livingstone, J. D. Winningham, and B. W. Reinisch, $F$-layer ionisation patches in the polar cap, J. Geophys. Res., 89, 1683, 1984.

Weber, E. J., J. A. Klobuchar, J. Buchau, H. C. Carlson Jr., R. C. Livingston, O. de la Beaujardiere, M. McReady, J. G. Moore, and G. J. Bishop, Polar cap $F$ layer patches: Structure and dynamics, J. Geophys. Res., 91, 12121, 1986.

Winningham, J. D., J. L. Burch, N. Eaker, V.A. Blevins, and R.A. Hoffman, The Low Altitude Plasma Instrument (LAPI), Space Sci. Instrum., 5, 465, 1981.

W. R. Coley and R. A. Heelis, William B. Hanson Center for Space Sciences, University of Texas at Dallas, P. O. Box 830688 M.S.Fo2.2, Richardson,TX75083-0688.(coley@utdallas.edu)

(Received May 15, 1995; revised August 2, 1995; accepted August 31, 1995.) 
